\title{
PROPELLANT BEHAVIOR IN LAUNCHER TANKS: AN OVERVIEW OF THE COMPERE PROGRAM
}

M. Dreyer

The paper gives an overview of the French-German research program COMPERE which is dedicated to the behavior of propellant in launcher tanks. The work logic follows the different flight phases of a launcher, i.e., the propulsive phase, engine shut-down, the ballistic phase, and the preparation of engine restart. Some examples of typical fluid motion are given for each flight phase. Performed benchmark tests are described and a sounding rocket experiment SOURCE planned for 2008 is mentioned.

\section{INTRODUCTION}

Reported herein are the accomplishments on a French-German research program on propellant behavior in launcher tanks (COMPortement des Ergols dans les REservoirs). The COMPERE group consist of members from industry (Air Liquide, Astrium ST, Cryospace), academia (IMFT, LEGI, ZARM), research organizations (ONERA) and agencies (CNES, DLR). The funding for this application oriented research program is provided evenly by the French Space Agency CNES and the German Aerospace Center DLR. The main objective of the COMPERE program is to improve the scientific knowledge concerning the fluid* motion occurring in a tank of a launcher during its flight. In the frame of COMPERE, the efforts are focused on the phenomena in relation with a ballistic phase of the flight including the transition between boosted and ballistic periods. Sloshing motions occurring during the propelled phase are addressed as well. Furthermore, the COMPERE program aims to provide models or correlations which allow taking into account these phenomena in the computational fluid dynamics (CFD) or engineering tools used by industrials in charge of launcher tank developments.

The critical points in the different flight phases have been identified by the COMPERE working group to be:

${ }^{*}$ Liquid and gas phases are defined as fluids. 
- Propulsive phase:

- sloshing, nonlinear effects, initiation of break-up;

- antislosh ring;

- formation of droplets;

- vaporization of droplets due to thermal effects or/and concentration;

- heat and mass transfer at interface and walls; and

- thermal stratification in draining phase.

- Engine shutdown:

- amplification of sloshing including geometric effects;

- heat and mass transfer at interface and walls;

- droplet and bubble formation by breakup;

- vaporization of droplets due to thermal effects or/and concentration; and

- disturbance forces and torques caused by liquid sloshing.

- Ballistic phase:

- location of the liquid within the tank, interface liquid/gas;

- characteristic time of damping for sloshing;

- heat and mass transfer at interface and walls;

- boiling, natural convection, Marangoni convection and thermal stratification; and

- dilution of gas into the liquid phase.

- Preparation of engine restart:

- geyser formation, liquid fragmentation, inclusion of gaseous bubbles, outgasing time, settling time;

- heat transfer at the wall in connection with fluid rising at the walls;

- heat and mass transfer due to depressurization and pressurization;

- draining for chilldown; and

- rising of bubbles due to chilldown.

These critical points together with a well-defined set of equations and boundary conditions help to guide the direction of the research. An overall emphasize is given to achieve the best similitude between tests (either on ground or in a compensated gravity environment) and the original situation of a large tank 
filled with cryogenic liquids (liquid hydrogen and liquid oxygen). Experimental and numerical studies have been involved to gain insight into these phenomena occurring in the different flight phases.

Several benchmarks have been performed to validate and improve CFD tools used by industry. Parabolic flights and drop tower tests have been performed to achieve a relevant environment for the test. Sounding rocket flight experiments are planned to observe problems with larger time scales in a microgravity environment.

This paper describes the work logic and illustrates some results ending with an outlook towards planned activities.

\section{PROGRAMMATIC STATUS}

Since its return to flight, the Ariane $5 \mathrm{ECA}^{*}$ has demonstrated a very good overall performance and serves as Europe's guaranteed access to space for medium and heavy payloads. Today's first priority is the stabilization and consolidation of Ariane 5 production and operation, which is realized within the $\mathrm{ACEP}^{\dagger}$ programme. This consolidation process includes, in particular, the exploitation of the currently used cryogenic upper stage ESC- $\mathrm{A}^{\ddagger}$, in order to maximize operational know-how in the field of cryogenic upper stages.

One of the most evident and best prepared evolutions of the Ariane 5 launcher system would be a second generation cryogenic upper stage, powered by a reignitable expander cycle engine. Such a new stage could lead to a larger performance and flexibility of the launcher as well as to a more robust and cost effective design. A thorough assessment of the institutional and market needs will help to define the future needs of the European launcher systems.

On the one hand, if these requirements point towards an increasing demand of performance and mission flexibility, an evolved cryogenic upper stage concept would turn out as an attractive means to reach these goals. On the other hand, the decision process for future evolutions of the existing launchers will also focus on the technical readiness level of the proposed scenarios. Clearly, any solution with a large benefit to the launcher system but with a limited development risk will receive a special consideration. In this sense, the activities within the FLPP and Ariane programmes, as well as the contents of COMPERE are a crucial tool to guide the decision process for future upper stage developments.

In the light of the long-term space transportation strategic plan, "universal" upper stage system concepts and technologies will be of particular interest, which allow easy transfer to other existing or future launcher systems. Although each

\footnotetext{
*Evolution Cryotechnique Type A.

${ }^{\dagger}$ Ariane Consolidation and Evolution Preparation.

${ }^{\ddagger}$ Etage Superieur Cryotechnique Type A.
} 
launcher requires its own designed architecture to maximize its performance, common elements like an expander cycle engine or know-how of cryogenic fluid management are essential technologies to focus on today.

\section{WORK LOGIC OF COMPERE}

The analysis of the known and anticipated flow phenomena is based on a generic view on a partly filled fuel tank, as depicted in Fig. 1. The characteristic dimensions of the tank are:

- tank height $0.5<H<3 \mathrm{~m}$;

- liquid depth $0.05 H<h<0.9 H ;$ and

- tank radius $0.7<R<2.7 \mathrm{~m}$.

The fuel tank may be subjected to accelerations $\mathbf{a}=10^{-5} g_{0}$ to $4 g_{0}$, where $g_{0}$ is the terrestrial gravity. The acceleration $\mathbf{a}$ is decomposed into a mean axial acceleration $\bar{a}$ acting on the fuel tank in $x$-direction (flight direction) and perturbation accelerations $a_{x}, a_{y}$, and $a_{z}$.

The tank is subjected to heat fluxes from the outer boundaries.

The tank contains two components (Fig. 1): propellant (or test liquid) which exists as a liquid phase or a gas phase (component 1), and the noncondensable pressurization gas which exists as a gas phase or dissolved in the propellant (component 2).

In normal operations, the tank contains two phases: a liquid phase of component 1 with component 2 dissolved in the propellant region, and a gas phase

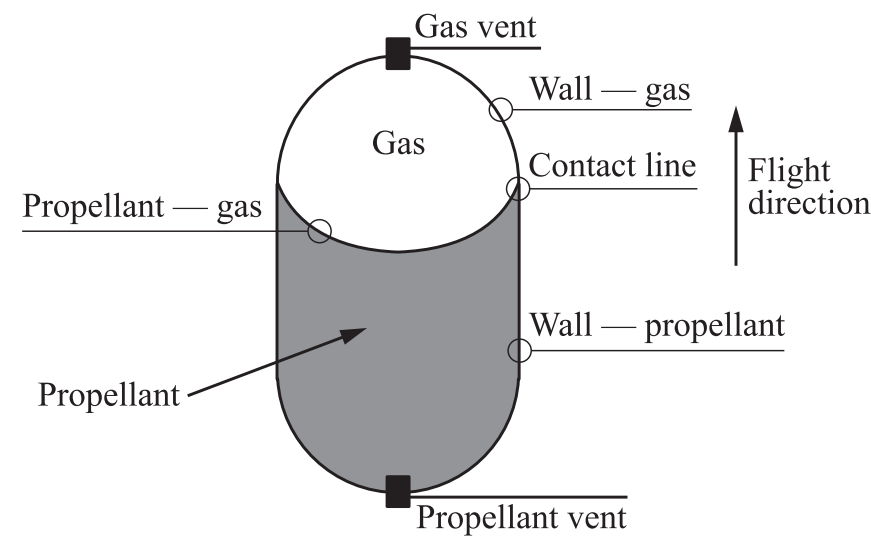

Figure 1 Schematic of a liquid propellant tank with different regions of interest 
as a mixture of components 1 and 2. At some flight conditions, violent sloshing may occur which creates a large amount of droplets in the ullage and/or large amount of bubbles in the propellant region.

The governing equations for the conservation of mass, linear momentum, and energy are formulated together with appropriate boundary conditions at the liquid-gas interface and at the wall. The scaling of these equations with characteristic values for velocity, time, pressure, and temperature leads to characteristic nondimensional numbers, which help to keep the number of tests down and to apply results from tests to the original situation. A certain set of dimensionless numbers has been identified for each flight phase. This helps to identify the relevant terms of the governing equations and to neglect terms of secondary importance.

\section{PROPELLED FLIGHT PHASE}

One of the critical points during the propelled phase is the excitation of sloshing due to lateral motion of the launcher. The resulting forces interfere with the attitude control system, thermal stratification is disturbed, and droplets can be produced depending on the amplitude of the resulting sloshing motion. Both pressure decrease and pressure increase can be the results.

A major propellant management task is to predict the oscillation amplitude and frequency as well as the effect on the temperature distribution and pressure changes. Sloshing at the tank scale during the propulsive phase is independent of surface tension and viscous effects except for the determination of damping coefficients and the investigation of wave breaking. Tests can therefore be performed on ground on a laboratory scale.

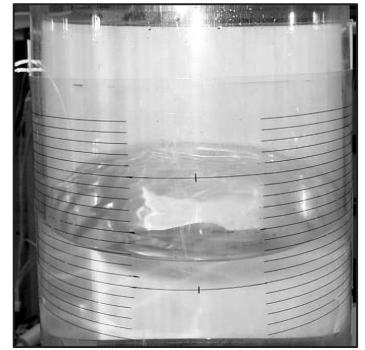

(a)

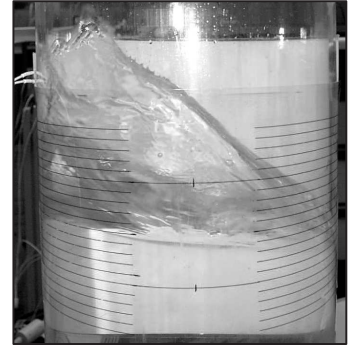

(b)

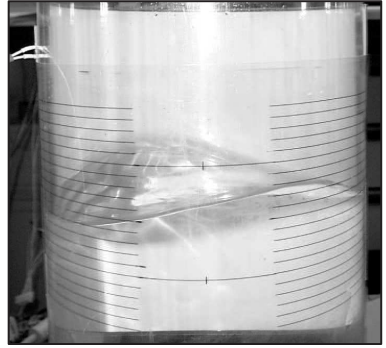

(c)

Figure 2 Different sloshing modes excited by lateral oscillations of a cylindrical tank: (a) first axisymmetric mode; (b) swirl mode; and (c) second symmetric mode. The direction of the tank motion is normal to the paper plane 
Experiments have been conducted by Air Liquide, Astrium ST, LEGI, and ZARM to investigate the different features of this flow phenomenon. The general aim is to validate the CFD codes used for the prediction of the sloshing motion. In particular, the interaction between the sloshing motion and the thermal behavior of the system must be known for avoiding disturbances or malfunctions during the flight.

To illustrate the phenomenon, some sloshing modes are depicted in Fig. 2. A sinusoidal excitation in the direction normal to the paper plane leads to different slosh modes; here, the first axisymmetric mode, the swirl mode, and the second symmetric mode.

The existence of the modes depends on the dimensionless frequency and amplitude. A more detailed paper on liquid sloshing and wave breaking in circular and square-base cylindrical containers has been published recently [1].

\section{BALLISTIC PHASE}

\subsection{Reorientation}

During the ballistic flight of an upper stage, the hydrostatic pressure reduces to very low values (depending on the residual acceleration of the stage) and capillary forces dominate the flow behavior. To simulate this situation, a cylinder partly filled with liquid was subjected to a sudden change in gravity during the free fall in the Bremen drop tower. The free surface moves from an essentially flat shape under normal gravity to a constant-curvature shape in microgravity conditions.

Figure 3 shows the evolution of the free surface. The features of this reorientation process for the isothermal case, such as initial and final shapes, initial rise
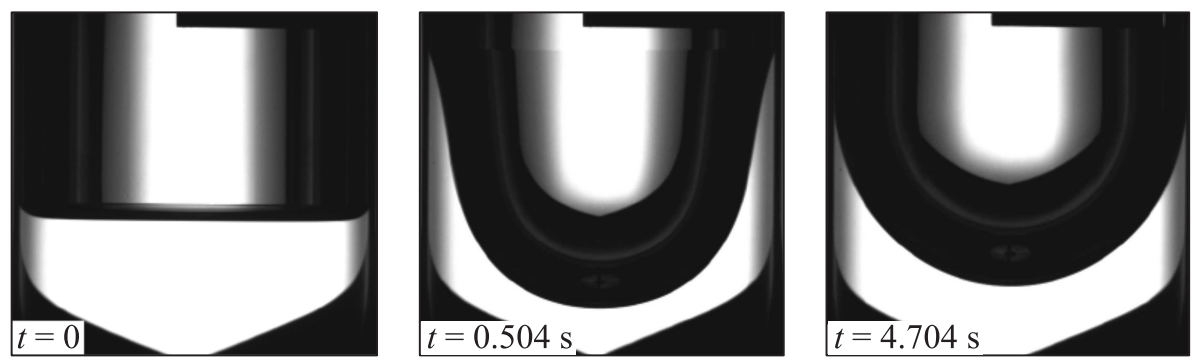

Figure 3 Series of video images showing the evolution of the free surface upon step reduction in gravity 

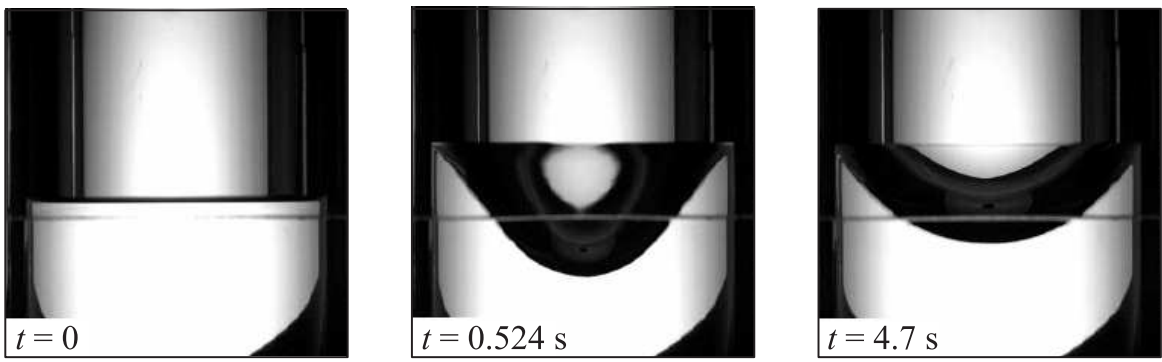

Figure 4 Series of video images showing the evolution of the free surface with a temperature gradient between upper wall and liquid

of the meniscus, frequency and damping of the oscillation, are described in [2]. The data have been used for the first COMPERE benchmark and a dedicated paper has been published [3].

The real situation is not isothermal since the heat fluxes from the ambient increase the temperature in the walls and in the ullage. In this case, during the reorientation (or axial sloshing mode) the cold liquid moves over a hot wall. If a noncondensable gas is present above the surface, a convection driven by a surface tension gradient sets in (Marangoni flow) and deforms the free surface in the vicinity of the contact line. This leads to a macroscopic change of the contact angle and influences the overall shape of the free surface.

The difference can be seen in Fig. 4, where the same liquid is now exposed to a temperature gradient. The features of the reorientation change and the final surface forms a nonzero contact angle with the wall even for this perfectly wetting liquid. This effect must be considered since the free-
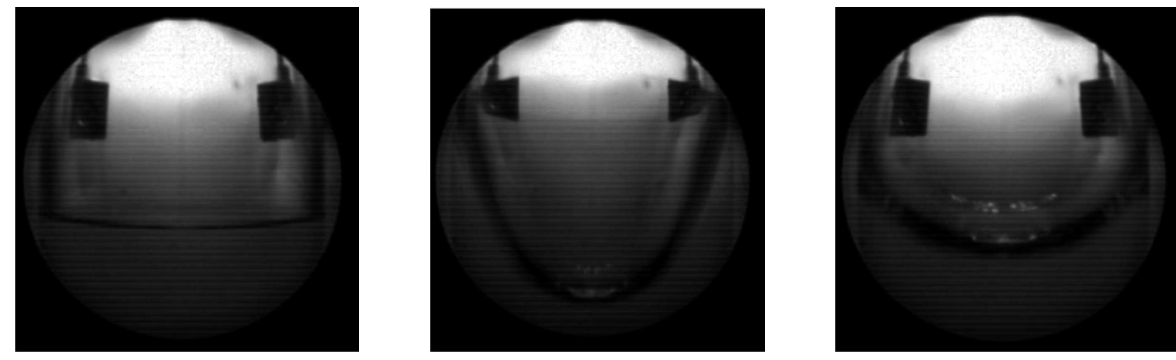

Figure 5 Series of video images showing the evolution of the free surface of liquid nitrogen upon step reduction in gravity. The images are taken through an endoscope and are therefore optically deformed 
surface shape plays a role in the heat and mass transfer inside a cryogenic tank.

Recently, the capabilities of performing experiments with cryogenic liquids have been extended at ZARM and tests with liquid nitrogen are underway. Figure 5 shows a series of video images with liquid nitrogen during the free fall in the Bremen drop tower. The experiment setup will be upgraded to enable tests with other cryogenic liquids like argon, neon, and methane, as well as nonisothermal boundary conditions in a cryogenic environment.

\subsection{Boiling}

During different phases of Ariane 5 missions (ballistic phases, ignition phases), it is important to know and to control the spatial distributions of the cryogenic fluid and of the pressurization gas in the tank. The variations of pressure and temperature strongly depend on the two-phase distributions as well as on interfacial and wall heat and mass transfer.

The first study performed at IMFT showed that the wall heat flux due to solar radiation should be sufficient for boiling incipience in microgravity conditions. Unfortunately, under these conditions, no predictive model does exist for the wall heat transfer prediction. Few experimental data are available and the correlations established in normal gravity cannot be extrapolated to microgravity conditions $[4,5]$.

The characteristic dimensionless numbers relevant to the study of heat transfer and phase change have been identified. To reach similar conditions in boiling for liquid oxygen and liquid hydrogen, the pressure of the test liquid (in this case, R123) has to be increased to 0.2 and $1 \mathrm{MPa}$, respectively. This imposes some constraints on the design of the test hardware for parabolic flights.

A research programme to investigate the boiling phenomena under microgravity has been defined and an experimental setup has been designed and built in order to determine:

- the relation between wall temperature and heat flux;

- the quantity of vapor generated and the evolution of the bubble layer thickness; and

- the temperature profile in the subcooled liquid.

Later on, many adaptations have improved the setup. The first experiments have been carried out during a parabolic flight campaign in March 2006. Figure 6 shows the growth of vapor bubbles during the ballistic phase of the A300 zero- $g$ aircraft. The test cell is equipped with thermocouples and video images are synchronized with the data acquisition system to enable the evaluation of the wall heat flux during the different phases of the bubble growth. 


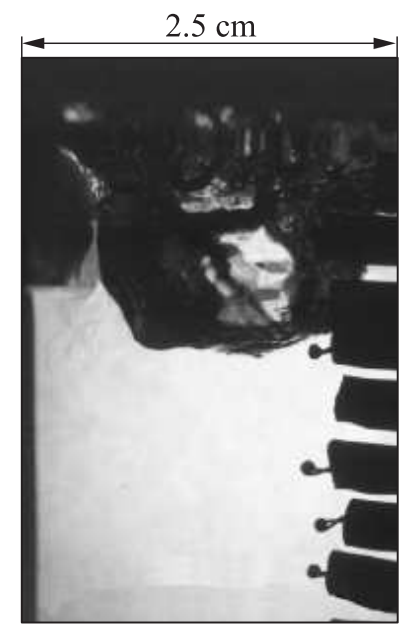

$t=113.2 \mathrm{~s}$

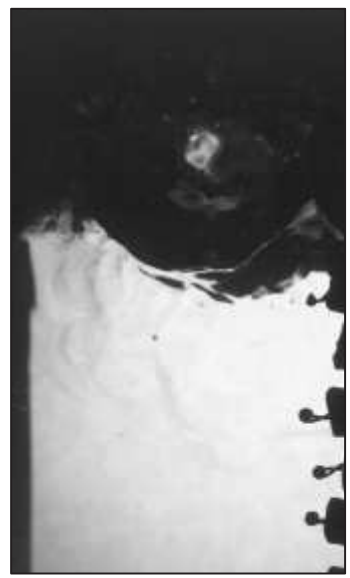

$t=115 \mathrm{~s}$

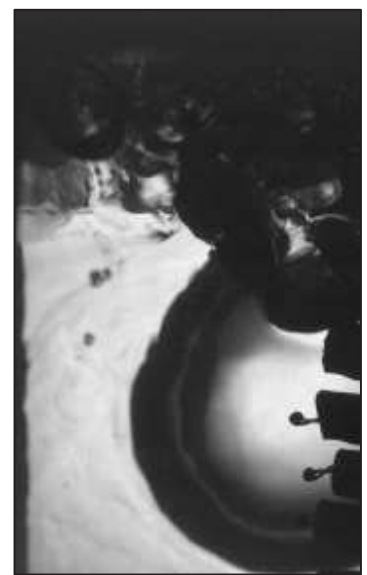

$t=118 \mathrm{~s}$

Figure 6 Series of video images showing the evolution of vapor bubbles during a parabolic flight

\section{PREPARATION OF RESTART}

\subsection{Geysering}

If the liquid is distributed in the tank as shown in subsection 5.1 and an axial thrust is applied to collect the propellant at the tank outlet, a geyser might be formed which creates a large amount of bubbles in the propellant phase. This phenomenon can be studied on ground by keeping the liquid away from the center of a cylindrical container by means of a sluice and then rapidly removing the sluice from the container. The liquid flows toward the center and forms a geyser whose height exceeds the initial surface height by an order of magnitude.

Figure 7 shows a laboratory experiment where the original initial condition of the liquid is simulated with a barrier. The results have been used for the second COMPERE benchmark.

The situation might be worse if the liquid would be located at the opposite side of the tank outlet due to some residual accelerations resulting from the drag on the stage. In this case, the way for the liquid to achieve momentum is much larger and the geyser might be more violent, creating bubbles in the liquid phase and even droplets in the gas phase. This flow problem has been analyzed in a recent publication [6]. 

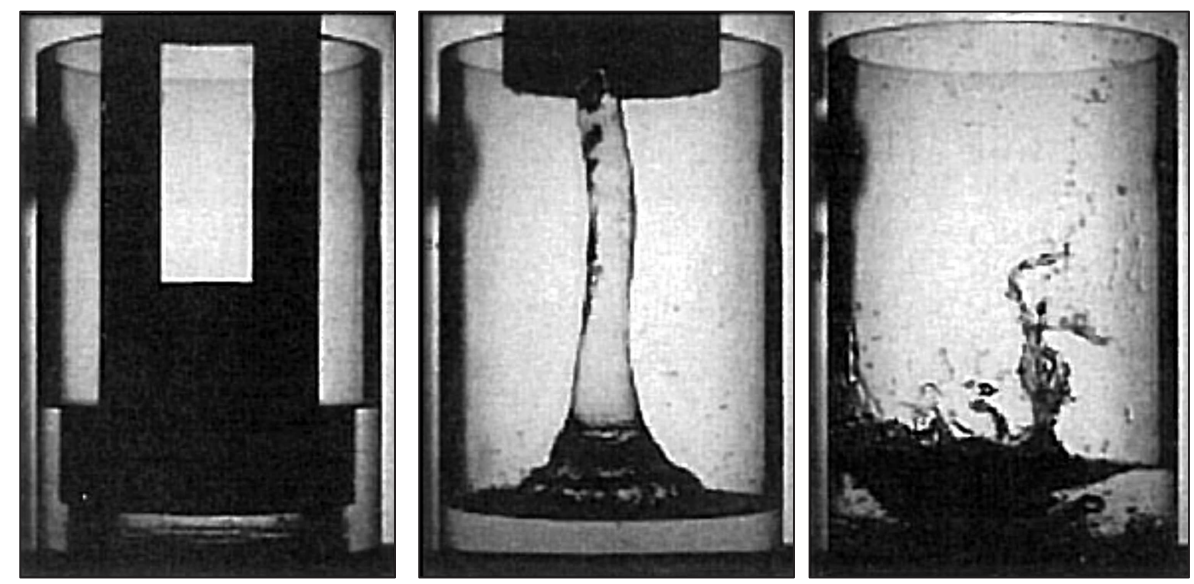

Figure 7 Series of video images showing the evolution of a geyser and the generation of bubbles in the bulk liquid

\subsection{Depressurization}

During different phases of the flight and, in particular, preparation of the restart of the engine, tank depressurization is necessary to keep the pressure in the allowed range and to condition the propellant, i.e., to decrease its temperature. Depressurization tests have, therefore, been performed by Air Liquide with different thermal stratification in the liquid and different depressurization rates.

Several tests have been performed using different ways of pressurization: pressurization due to thermal effects (self-pressurization), pressurization with hot (ambient temperature) gaseous $\mathrm{N}_{2}$, and no pressurization. Different outlet mass flow rates have been set by means of different orifices.

The strongest depressurization has been obtained for a saturated liquid (autopressurization) with a large depressurization rate (large exit diameter). With this strong depressurization, some droplets were created at the liquid surface and reached the top of the cryostat.

The evolution of the pressure can be divided into three phases:

(1) first phase with a very strong pressure variation;

(2) second phase with a quick pressure increase; and

(3) third phase with low pressure decrease.

The 2nd phase corresponds to the appearance of first bubbles. Then, pressure decrease (3rd phase) is accompanied by the creation of large gaseous pockets. 
All these depressurization cases are important to understand the behavior of cavitation depending on thermal stratification in the liquid and on the depressurization rate. The cases are difficult to be modeled due to a large amount of gaseous bubbles created.

Numerical analyses with respect to own depressurization experiments have been performed by Astrium ST. The goal of these analyses was to find an adequate way of modeling the depressurization process with available commercial software tools (FLOW-3D and FLUENT). The results of the described experiments are used as a benchmark experiment for comparison with the numerical models.

For both software tools, a mixture model was used. The model implies that bubbles are represented as a gas fraction within a numerical cell. Thus, free liquid/gas surfaces are characterized by strong gradients in the fluid fraction of the neighboring cells (from 1 for only liquid to 0 for only gas). The used model considered the liquid phase to be incompressible while compressibility was applied for the gas phase.

Comparisons between the experimental and numerical results were carried out. The numerical results showed that the general characteristic of the depressurization process can be modeled. It can be seen that evaporation occurs at the nucleation site, comparable to the experiment. However, after a while, gas bubbles were generated in the whole liquid volume. This is expected to be a consequence of the numerical model. Bubbles are only generated in cells which contain a nonzero amount of gas. If bubbles do not rise fast enough, then the neighboring cells in the liquid will see gas after a certain time. This results in a kind of foam layer if the bubble rise velocity is inadequate. This way, the bubbles will distribute from cell to cell since the whole liquid is overheated.

\section{BENCHMARK TESTS}

Two series of benchmark tests have been performed in the frame of the COMPERE program. The first series consisted of 4 test cases:

1. Sloshing in a rotating tank, subjected to sudden axial acceleration.

2. Lateral sloshing in a two-dimensional setup to study wave breaking.

3. Axial sloshing (reorientation) in an axisymmetric container after sudden reduction of gravity.

4. Stratification inside a closed liquid hydrogen tank.

Five commercial CFD codes have been tested, among them FLUENT and FLOW-3D. The computations have been performed partly by the code developers and partly by the end users in industry. The results have been analyzed by 
ONERA. As a consequence of the analysis, FLUENT and FLOW-3D have been chosen for the continuation of the benchmark, also mainly due to the fact that they are used by Air Liquide and Astrium ST.

The second series consisted of two test cases:

1. Liquid reorientation from high to low gravity conditions in an axisymmetric container, with heat transfer from the wall to the liquid. The test showed an appreciable effects of the temperature difference in the effective contact angle which should be captured by the CFD codes. The tests have been performed by ZARM in the Bremen drop tower.

2. Liquid reorientation from low to high gravity conditions, which creates a geyser. The experiments were performed by LEGI on ground.

Both test cases have been analyzed with FLUENT and FLOW-3D computations. The results not only helped the users to judge on the applicability of their codes, but also led to the implementation of submodels to cope with effects which cannot be modeled within meshes on the tank scale. A continuation of the benchmark is planned with regard to heat and mass transfer at the interface.

\section{ANTICIPATED FLIGHT EXPERIMENTS}

A sounding rocket experiment (SOURCE) for the Maser 11 mission is under development in the frame of an ESA MAP project in convective boiling and condensation (AO-2004-111). The objectives are listed below:

- to follow the effect of wall heat flux on the contact line and the free surface during the ballistic phase;

- to follow the boiling bubble behavior (growth, detachment, motion, and recondensation) on a local heater during the ballistic phase; and

- to follow the effect of depressurization at the end of the ballistic phase.

SOURCE is a benchmark type of experiment on fluid behavior in tanks to test hypotheses and numerical predictions (quantitative results on a tank scale).

A preheated transparent tank will be filled with liquid when the payload has entered the ballistic flight. The contact of the cold liquid with the hot tank wall in combination with the existence of a noncondensable gas causes a surface tension gradient driven flow (Marangoni flow) which deforms the free surface and increases the heat transfer to the liquid compared to a pure diffusive situation. This flow pattern will be observed for a while before boiling will be caused on a heater. Video downlink and telecommand uplink from the ground allows to change the heater power until bubbles are created. Their behavior 
in the compensated gravity environment of the ballistic phase will be studied. Before the end of the mission, the pressure in the tank will be reduced to create an overheated liquid and to generate bubbles again. This scenario has been chosen to achieve good similitude with an upper stage entering a ballistic phase. The results will be useful to generate models for heat and mass transfer in microgravity and to benchmark commercial CFD codes. The anticipated flight date is spring 2008 .

\section{OUTLOOK}

As a consequence of the actual state of knowledge, further actions have to be undertaken to allow the determination of pressure changes and liquid temperature stratification caused by liquid motion phenomena in the tank during different flight phases. The liquid motion phenomena are: sloshing, wave breaking, reorientation, capillary rise, geysering, boiling, and depressurization.

Flight experiments in a relevant environment with respect to future upper stages (repeated coasting phases of up to $5 \mathrm{~h}$ with engine reignition) are needed to cover the following critical points: characteristic time of damping for sloshing, heat and mass transfer at interface and walls, boiling, Marangoni convection and thermal stratification, and dilution of gas into the liquid phase. The sounding rocket COMPERE experiment (SOURCE) is a first step in this direction.

\section{ACKNOWLEDGMENTS}

The funding of the German research projects by the German Ministry of Education and Research (BMBF) through the German Aerospace Center (DLR) under grant numbers 50JR0011 and 50RL0741 is gratefully acknowledged. The funding of the French research projects by the CNES directorate of launcher is gratefully acknowledged. The author acknowledges the input received for this paper by references below.

\section{REFERENCES}

1. Royon-Lebeaud, A., E. J. Hopfinger, and A. Cartellier. 2007. Liquid sloshing and wave breaking in circular and square-base cylindrical containers. J. Fluid Mech. 577:467-94.

2. Dreyer, M. 2007. Free surface flows under compensated gravity conditions. Springer Tracts in modern physics ser. No. 221. Berlin-Heidelberg: Springer. 
3. Michaelis, M., and M. E. Dreyer. 2004. Test-case No 31: Reorientation of a free liquid interface in a partly filled right circular cylinder upon gravity step reduction (PE). Multiphase Sci. Technol. 16(1-3):219-38.

4. Lee, H.S., and H. Merte, Jr. 1996. Hemispherical vapor bubble growth in microgravity: Experiments and model. Int. J. Heat Mass Transfer 39(12):2449-61.

5. Straub, J. 2001. Boiling heat transfer and bubble dynamics in microgravity. In: Advances in heat transfer. Eds. J. Hartnett, T. Irvine, Y. Cho, and G. Greene. Academic Press. 35:57-172.

6. Baumbach, V., E. J. Hopfinger, and A. Cartellier. 2005. The transient behaviour of a large bubble in a vertical tube. J. Fluid Mech. 524:131-42. 